\title{
Improved Weighted Page Ranking Algorithm Based on Principal Component Analysis and Map Reduce Frame work for Web Access
}

\author{
T. Mylsami ${ }^{1}$ and B. L. Shivakumar ${ }^{2}$ \\ ${ }^{1}$ Department of Computer Science and Information Technology, \\ Dr. G.R. Damodaran College of Science, Coimbatore, Tamil Nadu, India \\ ${ }^{2}$ Principal, Sri Ramakrishna Polytechnic College, Coimbatore, Tamil Nadu, India \\ E-Mail: mylsamigrd@gmail.com
}

\begin{abstract}
In general the World Wide Webbecome the most useful information resource used for information retrievals and knowledge discoveries. But the Information on Webto be expand in size and density. The retrieval of the required information on the web is efficiently and effectively to be challenge one. For the tremendous growth of the web has created challenges for the search engine technology. Web mining is an area in which applies data mining techniques to deal the requirements. The following are the popular Web Mining algorithms, such as PageRanking (PR), Weighted PageRanking (WPR) and Hyperlink-Induced Topic Search (HITS), are quite commonly used algorithm to sort out and rank the search results. In among the page ranking algorithm uses web structure mining and web content mining to estimate the relevancy of a web site and not to deal the scalability problem and also visits of inlinks and outlinks of the pages. In recent days to access fast and efficient page ranking algorithm for webpage retrieval remains as a challenging. This paper proposed a new improved WPR algorithm which uses a Principal Component Analysis technique called (PWPR) based on mean value of page ranks. The proposed PWPR algorithm takes into account the importance of both the number of visits of inlinks and outlinks of the pages and distributes rank scores based on the popularity of the pages. The weight values of the pages is computed from the inlinks and outlinks with their mean values. But in PWPR method new data and updates are constantly arriving, the results of data mining applications become stale and obsolete over time. To solve this problem is a MapReduce (MR) framework is promising approach to refreshing mining results for mining big data .The proposed MRalgorithm reduces the time complexity of the PWPR algorithm by reducing the number of iterationsto reach a convergence point.
\end{abstract}

Keywords: Information Retrieval (IR), Search Engine, Hyperlinks, Elements, Page Ranking (PR), Principal Component Analysis (PCA), Map Reduce (MR) Framemwork, Weighed page Ranking (WPR)

\section{INTRODUCTION}

The growth of information over the internet is huge and the World Wide Web (WWW) has become a more useful environment to storeand retrieve information. Due to growth of WWW increases the difficulty of dealing information from different perception of knowledge seekers such as business analysts and web service providers [1]. Nowadays retreiving of web pages based on user query words is not a big issues in search engine [2], instead the challenege is that a search engine will return a large number of web pages to user queries and user have to spend more time in searching relevant information fromlist resulting in information overload problem for accessing [3-5]. Normally the users use information retrieval tools like search engines to find information from the World WideWeb. There are more than hundreds of search engines are available in usage, some are popular like Google, Yahoo, Bing etc., are strong in crawling and ranking methodologies. Usually the search engines download, index and store hundreds of millions of web pages and responsing the millions of queries every day. So the Web mining and ranking mechanism becomes very vital for effective information retrieval in Web.

Among the various search engine, Google is very successful because of its Page Rank algorithm. The search engine with Page ranking algorithm will gives the search results based on the relevance, importance and content score and Web mining techniques is used to order them based on user interest.In few Ranking algorithms depend only on the link structure of the documents i.e. based on the popularity scores (web structure mining), but the other apprear for the actual content in the documents (web content mining), and others will use a combination of both i.e. they use content of the document as well as the link structure to assign a rank value for a given document in web. But the search engine respone are not based on the user interest, so the engine will loose its nature and popularity in web. The following are few Page rankign algorithm are discusedd as follows:

Weighted Page Rank [6] Algorithm is proposed by Xing and Ghorbani Wenpu Xing and Ali Ghorbani. Weighted Page Rank algorithm (WPR) is the modification of the original page rank algorithm. WPR decides the rank score based on the popularity of the web pages by taking into consideration and it uses both the in-links and out-links of the pages. This algorithm concept generates high value of rank to the more popular webpages and but not equally divide the rank of a webpage among its out-link webpages. It consider higher accuracy in terms of ranking because it uses the content of the webpages. This algorithm strongly consider the popularity of the web page.

Distance rank algrithm called as an intelligent ranking algorithmproposed by Bidoki and Yazdani [7]. This 
algorithm is based on reinforcement learning algorithm. The key characteristics of this algorithm is that the distance between pages is considered as a punishment factor.This algorithm uses the ranking is done on the basis of the shortest logarithmic distance between two pages and ranked according to them. The key advantage of distance rank algorithm to find pages with high quality and more quickly with the use of distance based solution. The drawback of distance rank algorithm is that the crawler should perform a large calculation to calculatethe distance vector, if new page is inserted between the two pages.

Abou-Assaleh et al., [8] introduced a focused surfer model and ranking method for improving search results for focused search paradigms. This model is shown to achieve significant performance and accuracy benefits over vanilla PageRank and approximately equivalent accuracy as TopicSensitive Page Rank without the online processing overhead. The major disadvantages of this ranking model is that if the topics have subtopics and documents belong to these with changing probabilities, relationship of topic membership and transmission of rank is a complicated task in this method.

Lamberti et al., [9] proposed a relation based algorithm for the ranking the web page for semantic web search engine. Different search engines are existing for better information extraction by using relations of the semantic web. This relation based page rank algorithm for semantic web search engine that depends on information extracted from the queries of the users and annotated resources. Among th various methods specfically the results are very encouraging on the constraint of time complexity and accuracy. The key updation of this algorithm to increase the use of scalability into future semantic web repositories. Normally in this ranking algorithm every webpage is to be annotated with respect to some ontology, which is the very tough task.

Lee et al., [10] propose a novel framework of querydependent ranking. The simple likeness measure is used to calculate similarities between queries. This algorithm uses a process of creating an individual ranking modelfor each training query with resultant documents. A new query is raised by the user, documents retrieved for the new query is ranked based on the certain scoresby a ranking model and which is joint from the models of similar training queries. Then a method is used for formative combining weights is also provided. The limitation of this method it uses only the inadequate numbers of characteristics to calculate the similarity.

Vojnovic et al., [11] have proposed a ranking and suggestive algorithm for popular items based on user feedback. Then the user feedback is calcul;ated using a set of recommended items and also items are selected based on user preferences. The propose of this methodto measure the correct ranking of the items based on the actual and unbiased popularity. In the proposed algorithm has various tmethods for suggesting the search query. This algorithm used for providing tag submission for social tagging system and not considering substitute user choice model for alternative rules for ranking andsuggestive rules.

Cheng et al., [12] have used page rank and HITS algorithm used for ranking. The key advantages, drawbacks and possibility of different algorithms used in web are accessed for ranking the journal.Impact factor is a very popular for ranking the journal but it has basicdrawbacks for the ranking is based on counting the in degrees of the nodes in the collection and not considering the impact of prestige of the journal in which the citations are present.

Weighted Page Content Algorithm (WPCA) [13] is the change of exclusive page rank algorithm. This algorithmspecfically used to deliver the sorted order of the webpage. The unique feature of WPCR [13] uses a numerical value based on the webpage as given in order. This method uses both Web struture mining and Web content mining. The web structure mining is used to examine the webpage popularities and then Web content is to calculate the page relevancy. This algorithm consider both the in links and out links for calculation purpose [14].

Hyperlink Induced Topic Search (HITS) is the most reliable and extensively used for personalized ranking algorithm on all networks. The HITS algorithm computer ranks nodes on netwroks based on power iteration and high difficulity of calculating values.The HITS algorithm with the Monte Carlo method [15], and proposes Monte Carlo used for HITS computation. From the theoretical analysis and experiments will produce the Monte Carlo based rough computing of the HITS ranking and it reduces computing resources a lot while maintenance of higher accuracy value, and this methodconsiderably better than exiting works. It conclude that HITS based algorithm will not solve the solve scalability problem in any possible way.

The Weighted Page Rank used to contains both in link and out link of the webpage and the link is assigned based on the page rank precedence. The terms [16] of weight values to the incoming and outgoing links and are denoted as $w^{\text {in }}(m, n)$ and $w^{\text {out }}(m, n)$ respectively, the weight of link $(m, n)$ calculation depends on the number of in links of page $\mathrm{n}$ and as well the number of in links of all reference pages of page $\mathrm{m}$. This method of work the scalability problem is not sorted and the weight values of these links is optimized through the Principal Compoenet Analysis (PCA) in this work. The major contribution of the work is extended from this WPR schema.

\section{PROPOSED WEIGHTED PAGERANKING AND MAP REDUCE FRAMEMWORK}

In general the growth of the Internet data in Web is huge and users get easily missing in the rich hyper structure of the web. The primary objective of website design is that to give the required information to the users in time. The key objective of the user requirement is to reach the correct web 
content in posible time constriant. To over come the user needs, Web mining is used to classify users and web pages by predicting the users' behavior and the order of the URLs be likely to be accessed in order. Web structure mining plays an eminent role in this approach for various tasks. Usually the two page ranking algorithms like HyperlinkInduced Topic Search (HITS) and Page Ranking (PR)are widley used in web structure mining. Among both algorithms will take care of all links equally when distributing rank scores. Among the frequent algorithms have been developed to improve the performance of these methods in possible way. The functions of Weighted Page Ranking algorithm (WPR) [16], an extension to the standard PageRanking algorithm [17]. The key functions of WPR takes into consideration of both the inlinks and the outlinksof the pages and then it distributes the rank scores among the popularity of the webpages. Actually the WPR method works on the standard is that if a page is important, many linkages from other web pages to it or are linked to it. In WPR uses the every node does not have an equal chance to get visited by the random surfer method, but in some siutation nodes with high weights will get high chance. In WPR algorithm some nodes have high weights values has been assigned and but even the webpage is not importantit display for consideration, to solve this problem statement this researh work WPR is combined to Principal Component Analysis (PCA) and named as Principal Weighted Page Ranking (PWPR) algorithm and another key challenge in web mining is that new data and updates are continuously arriving in webpage and the results of data mining applications become tough and outdated over time. To overcome this problem after the web pages are ranked it is represented as the graph model. Even though in graph modelthe number of connections is high the launch of graph model is difficult for larger samples cases. To solve this problem web page ranked method is to reduce challenge by a new model without changing the propertiesby using Map Reduce (MR) Framework see Fig.1 for overall represenation.

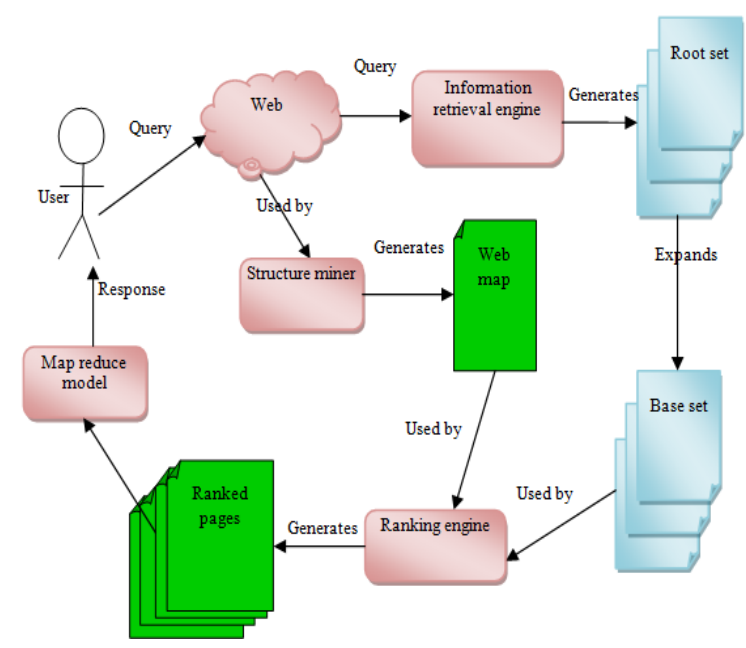

Fig. 1 Architectural representation for PWPR algorithm

\section{A. Principal Weighted Page Ranking (PWPR)}

In general the Weighted Page Ranking (WPR) algorithm is an expansion of the Page Ranking algorithm. The key functions of this algorithm assigns a larger rank values to the more important pages instead of dividing the rank value of a webpage equally between its outgoing linked web pages. In this algorithm each outgoing link gets a value proportional to its importance and it is assigned in terms of weight values to the incoming and outgoing links. The web page rank is calculated using the following methods.

$$
\begin{aligned}
& W P R_{V O L}(u)= \\
& (1-d)+d \sum_{v \in B(u)} W P R_{V O L}(v) W^{\text {in }}(v, u) W_{V O L}^{\text {out }}(v, u)
\end{aligned}
$$

Where $W P R_{V O L}(u)$ and $W P R_{V O L}(v)$ represent page rank of web page ' $u$ ' and ' $v$ ' respectively, ' $d$ ' is the dampening factor, $\mathrm{B}(\mathrm{u})$ is the set of web pages pointing to $\mathrm{u}, L_{u}$ is number of visits of links pointing from $\mathrm{v}$ to $\mathrm{u}, T L(v)$ is the total number of visits of all links from $\mathrm{v}, W^{i n}(v, u)$ represents the reputation from the number of inlinks of $u$. Then it is calculated based on the number of incoming links of page $u$ and the number of incoming links of all reference pages of page $\mathrm{v}$.

$$
W^{i n}(v, u)=\frac{I_{u}}{\sum_{p \in R(v)} I_{P}}
$$

$I_{u}$ is number of incoming links of page $\mathrm{n}, I_{P}$ is number of incoming links of page $\mathrm{p}, R(v)$ is the reference page list ofpae $v$.

$$
W_{V O L}^{\text {out }}(v, u)=\frac{o_{u}}{\sum_{p \in R(v)} O_{P}}
$$

Where $O_{u}$ and $O_{P}$ represents the outgoing visits of links of page $u$ and $p$ respectively and $R(v)$ represents the set of reference pages of page v. From this functions are determined by using the above two equation (2) and equation (3) and it values still need to optimize to increase web page rank results. So through the proposed work Principal Component Analysis (PCA) technique is introduced to find new $W^{\text {in }}(v, u), W_{V O L}^{\text {out }}(v, u)$ values by calculating covariance values.

\section{B. Principal Component Analysis (PCA) for Weight Updating}

The Principal Component Analysis (PCA) [18] is a mathematical procedure intended to replace a number of correlated visit of link weight of out and inline links with a new set of weight values that are linearly uncorrelated. The preparation of weight values in web pages is done by altering of variables given by an orthogonal transformation. The technique is well known for mathematical calculation. Here the simple approach with two dimensions for weight values computations consider for both in link and out link web pages. Let consider two dimensional points $\left(x_{1,1}^{\text {in }}, y_{1,1}^{\text {out }}\right),\left(x_{2,1}^{\text {in }}, y_{2,1}^{\text {out }}\right), \ldots,\left(x_{u, v}^{\text {in }}, y_{u, v}^{\text {out }}\right)$ such as inlink and 
outlink webpages. Through the correlation between the $x_{i, j}^{i n}$ and the $y_{i, j}^{\text {out }}$ can be calculated by their variance

$$
\begin{gathered}
\sigma_{x y}=\left(x^{\text {in }}-\bar{x}^{\text {in }}\right)\left(y^{\text {out }}-\bar{y}^{\text {out }}\right), \text { where, } \\
\bar{x}^{\text {in }}=\sum_{u=1}^{n} \sum_{v=1}^{m} x_{u, v}^{\text {in }} \\
\bar{y}^{\text {out }}=\sum_{u=1}^{n} \sum_{v=1}^{m} x_{u, v}^{\text {in }}
\end{gathered}
$$

represents the average value of $x_{u, v}^{i n}$. Let $\mathrm{X}$ is the visited web pages link information matrix whose columns are the original coordinates of the given points:

$$
X=\left(\begin{array}{cccc}
x_{1,1}^{\text {in }}, y_{1,1}^{\text {out }} & x_{2,1}^{\text {in }}, y_{2,1}^{\text {out }} & \ldots & x_{n, 1}^{\text {in }}, y_{n, 1}^{\text {out }} \\
x_{1,2}^{\text {in }}, y_{1,2}^{\text {out }} & x_{2,2}^{\text {in }}, y_{2,2}^{\text {out }} & \cdots & x_{n, 2}^{\text {in }}, y_{n, 2}^{\text {out }} \\
\vdots & \vdots & \ddots & \vdots \\
x_{1, m}^{\text {in }}, y_{1, m}^{\text {out }} & \cdots & \vdots & x_{n, m}^{\text {in }}, y_{n, m}^{\text {out }}
\end{array}\right)
$$

and $\mathrm{X}^{\prime}$ is the analogous matrix with the new coordinates:

$$
\mathrm{X}^{\prime}=\left(\begin{array}{cccc}
x_{1,1}^{\text {in }}, y_{1,1}^{\text {out }} & x_{2,1}^{\text {in }}, y_{2,1}^{\text {out }} & \cdots & x_{n, 1}^{\text {in }}, y_{n, 1}^{\text {out }} \\
x_{1,2}^{\text {in }}, y_{1,2}^{\text {out }} & x_{2,2}^{\text {in }}, y_{2,2}^{\text {out }} & \cdots & x_{n, 2}^{\text {in }}, y_{n, 2}^{\text {out }} \\
\vdots & \vdots & \ddots & \vdots \\
x_{1, m}^{\text {in }}, y_{1, m}^{\text {out }} & \cdots & \vdots & x_{n, m}^{\text {out }}, y_{n, m}^{\text {out }}
\end{array}\right)
$$

The correlation matrix of $\mathrm{X}$ is

$$
\begin{gathered}
C=\frac{1}{n m} X \mathrm{X}^{\prime}=\left(\begin{array}{cc}
\sigma_{x}^{2} & \sigma_{x y} \\
\sigma_{x y} & \sigma_{y}^{2}
\end{array}\right) \\
C^{\prime}=\frac{1}{n m} X^{\mathrm{X}^{\prime \mathrm{T}}}=\left(\begin{array}{cc}
\sigma_{x^{\prime}}^{2} & \sigma_{x^{\prime} y^{\prime}} \\
\sigma_{x^{\prime} y^{\prime}} & \sigma_{y^{\prime}}^{2}
\end{array}\right)
\end{gathered}
$$

From this Eigen values of $\mathrm{C}$ are the roots of its characteristic polynomial

$\left|\begin{array}{cc}\sigma_{x}^{2}-\lambda & \sigma_{x y} \\ \sigma_{x y} & \sigma_{y}^{2}-\lambda\end{array}\right|=\lambda^{2}-\left(\sigma_{x}^{2}+\sigma_{y}^{2}\right) \lambda-\sigma_{x}^{2} \sigma_{y}^{2}-\sigma_{x y}^{2}$

The roots can be found with the usual formula to solve second degree equations:

$\lambda=\frac{\sigma_{x}^{2}+\sigma_{y}^{2} \pm \sqrt{\left(\sigma_{x}^{2}+\sigma_{x}^{2}\right)^{2}-4 \sigma_{x}^{2} \sigma_{y}^{2}-4 \sigma_{x y}^{2}}}{2}$

$=\frac{\sigma_{x}^{2}+\sigma_{y}^{2} \pm \sqrt{\left(\sigma_{x}^{2}-\sigma_{x}^{2}\right)^{2}-4 \sigma_{x y}^{2}}}{2}$

Let $\lambda_{+}$be the root obtained using the plus sign in the formula, and $\lambda_{-}$the one obtained with the minus sign. Then it can be easily checked that the following are Eigenvectors verifyingCVar${ }_{ \pm}=\lambda_{ \pm} \operatorname{Var}_{ \pm}$.
$\operatorname{Var}_{+}=\left(\begin{array}{l}\sigma_{x}^{2}+\sigma_{x y}-\lambda_{-} \\ \sigma_{y}^{2}+\sigma_{x y}-\lambda_{-}\end{array}\right), \operatorname{Var}_{-}=\left(\begin{array}{c}\sigma_{x}^{2}+\sigma_{x y}-\lambda_{+} \\ \sigma_{y}^{2}+\sigma_{x y}-\lambda_{+}\end{array}\right)$

Now the equation (1) is updated by using PCA, then the rank of web page can be calculated as given as follows.

$W P R_{V O L}(u)=(1-d)+d \sum_{v \in B(u)} W P R_{V O L}(v) C$

Where $W P R_{V O L}(u)$ and $W P R_{V O L}(v)$ represent page rank of web page ' $u$ ' and ' $v$ ' respectively, ' $d$ ' is the dampening factor, $\mathrm{B}(\mathrm{u})$ is the set of web pages pointing to $\mathrm{u}$.

\section{Principal Weighted Page Ranking (PWPR) Algorithm}

1. Finding a website: The website with rich hyperlinks is to be selected depends on the hyper structure of website.

2. Create graph model: For selected website, web graph $G$ a generated in which nodes represent web pagesand edges represent hyperlinks between web pages.

3. Calculating number of visits of hyperlinks: Client side script is used to monitor the hits of hyperlinks and information is sent to the web server and this information is accessed by crawlers.

4. Calculate page rank of each web page: The values of $\mathrm{W}_{\mathrm{VOL}}^{\text {in }}(\mathrm{v}, \mathrm{u})$, the popularity from the number of visits of in links and $\mathrm{W}_{\mathrm{VOL}}^{\text {out }}(\mathrm{v}, \mathrm{u})$, the popularity from the number of visits of out links are calculated for each node using formulae given in equation 8 and 9 and these values are substituted in equation 13 to calculate values of page rank.

5. Repetition of step 4: The step 4 is used recursively until a stable value of page rank is obtained.

\section{Map Reduce (MR) Framework}

After initializing all ranking scores, the computation performs a Map Reduce (MR) Frame work [19-20] per iteration to reduce the computation complexity of the web page rank model in G. Let us consider two Map Reduce jobs Jand $J$ 'performingthe same computation on input data set $D$ and $D^{\prime}$ respectively. $D^{\prime}=D+\Delta D$, where $\Delta D$ consists of theinserted and deleted input web page dataset with rank values for $\left\langle K_{1}, V_{1}\right\rangle s 1$. An update canbe represented as a deletion followed by an insertion.

The key objective of this work is to re-compute only the Map and Reduce function call and web page ranking instances that are affected by $\Delta D$ and the Incremental calculation for Map is straight forward. Simply invoke the Map function for the inserted or deleted $\left(\mathrm{K}_{1}, \mathrm{~V}_{1}\right)$. Then the other input $\mathrm{KV}$ pairs are not changed, their Map computation would remain the same. Computed the delta intermediate values, denoted $\Delta \mathrm{M}$, including inserted and deleted $\left(\mathrm{K}_{2}, \mathrm{~V}_{2}\right)$. 


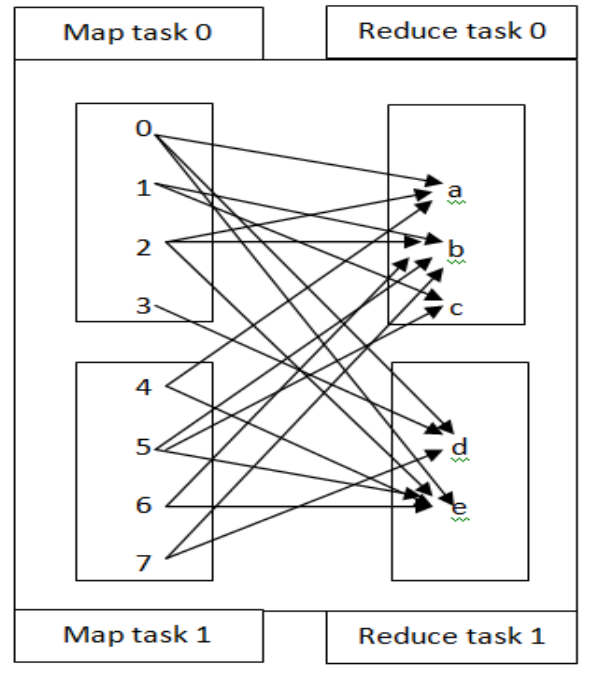

Fig. 2 Map Reduce Framework

Every edge contains three pieces of information: (i) the source Map instance, (ii) the destination Reduce instance, and (iii) the edge value. Map Reduce job is executed in two main functions of user defined data conversion, namely, map and reduce. When a jobs $\mathrm{J}$ is launched from the web page ranking graph model, the number of input page data is split into physical blocks and then distributed to cluster nodes for the parallel and distributed processing of the job task. Each web page rank block is viewed as a list of key value pairs $\left(\mathrm{K}_{1}, \mathrm{~V}_{1}\right.$. $)$ Here the key represents the page, $\mathrm{K}_{1}$ and $\mathrm{V}_{1}$ contains " $j_{1}: w_{i, j_{1}} ; j_{2}: w_{i, j_{2}} ; \ldots$ " where $\mathrm{j}$ is a target vertex and $w_{i ; j}$ is the weight of out-edge $(i ; j)$.

The rank values across for web pages of all nodes, which are connected with the same key, are grouped together and then forward as input to the reducer component called reduce. Each reducer executes the reduced graph model with correct rank value and generates a third set of key value pairs considered as the output of the job. In addition in the proposed framework master key value is also added to key pair for identifying the next key node in the graph model. The mapper receives the rank value (WPR vol (u)) for each user and outputs a key-value pair $\left(\mathrm{K}_{1}, \mathrm{~V}_{1}\right)$ based on the rank value. Since all the values related with the same key (i.e., partition number) are sent to a single reducer by the Map Reduce and the reducer can find all nearest features of reference objects in a partition area without any omitted. Finally the reducer function makes the predicate new graph model along with their page data information as $\in$ new $\mathrm{WPR}_{\mathrm{VOL}} \quad(\mathrm{u})$ of $\left(W P R_{V O L}(u)_{e}\right)$ with distinct predicate sets in the value list.

The new mapped web page rank model outputs new key value $\operatorname{pair}\left\langle K_{1}=\right.$ nodeposition, $M K_{1}=W P R_{V O L}(u)_{f}$, $\left.V_{1}=W P R_{V O L}(u)_{e}\right\rangle$, whereWPR $R_{V O L}(u)_{e}$ is a rank value of the page and $W P R_{V O L}(u)_{f}$ of is its neighboring rank value in the graph model.The second phase conducts the first update stage of finding all retained sets and some emerging sets through "one" MapReduce job. When each mapper web page instance starts, the mapper first builds a group of candidates with the previous key value pair and border sets information cached. When the mapper is fed with the remainsof new graph model, i.e., the output of the phase-1, $\left\langle K_{1}=\right.$ nodeposition, $M K_{1}=W P R_{V O L}(u)_{f}, V_{1}=$

newWP $\left.R_{V O L}(u)\right\rangle$, the map function reads newWPR $R_{V O L}(u)$, and outputs candidate instance sets in newWP $R_{V O L}(u)$ with value $=1$.

In the final stage Reduce function computes for a vertex $\mathrm{j}$ the sum of all its in-edge weights as $\sum_{\mathrm{i}} w i, j$. The example of the reduced function is shown in Fig. 3 shows the delta input for the updated application graph. A ' + ' symbol indicates a newly inserted kv-pair, while a ' - ' symbol indicates a deleted kv-pair and an update is represented as a deletion followed by an insertion.

For example, the deletion of vertex 1 and its edge are reflected as $\left\langle 1 ; 2: 0: 4 ;^{\prime}-{ }^{\top}\right\rangle$. The insertion of vertex 3 and its edge leads to $\left\langle 3 ; 0: 0: 2 ;{ }^{\prime}+'\right\rangle$. The modification of the vertex 0 's edges are reflected by a deletion of the old record $\left\langle 0 ; 1: 0: 2 ; 2: 0: 2 ;^{\prime}-{ }^{\prime}\right\rangle$ and an insertion of a new record $\langle 0$; $\left.2: 0: 4 ;{ }^{\prime}-'\right\rangle$.
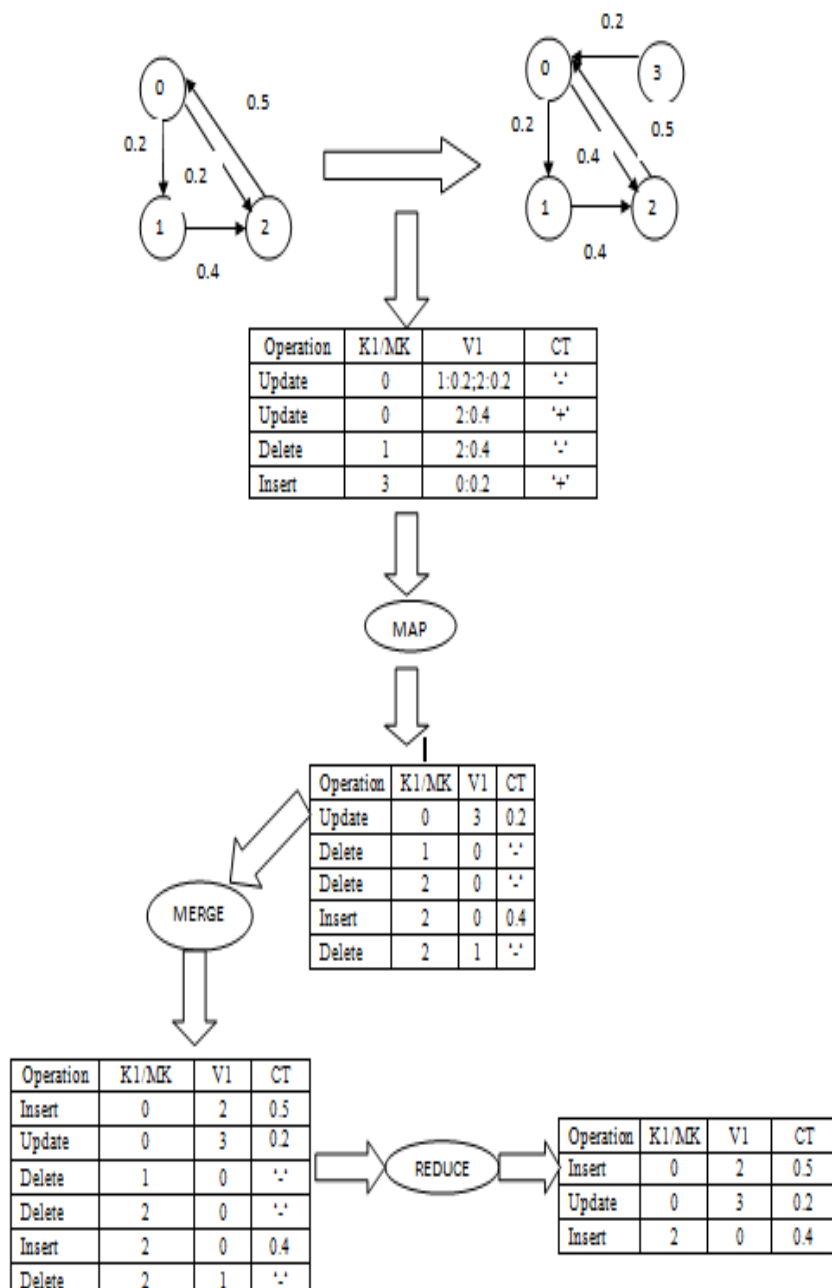

Fig. 3 Example of MR framework 


\section{EXPERIMENTATION RESULTS}

In this section experimentation result of the page raking algorithms such as PR, WPR, EWPR HITS and proposed PWPR algorithm is implemented on a Data set of 5000 URLS. Thus the URL'S has been composed from different categories (ref. Alexa.com). The execution part has divide in to three phases. Phase-1: To do Calculation of Users interest. Phase-2: Growth analysis Rate and Phase-3: Assigning Ranking for Web Pages using Users Interest.

\section{Phase-1}

A. Calculation of Users interest (I): User interest (I) calculated using 5000 URLS that are collected and hosted on goongo server named www.goongo.in. Goongo was popularized by using social media and oral advertising. For the time stamp of 15 days has been taken in to the account. The user's interest for every 15 days of usage of Goongo was observed [21] between two search engines google and Yahoo.

\section{Phase-2}

B. Growth analysis Rate: From the pahse2 the growth analysis rates of the web page is fetch and carry from the web information site "alexa.com [21]. From the value of each web page exist on the dataset is collected from the web site. These values are assigned to the web page based on various facts like network traffic analysis, number of distinct users of the site, average time spent by the user on the web page etc... This value can be positive or negative. It is purely dependent on its value versus the previous three months. Considering the above factors the growth analysis can be concluded.

\section{Phase-3}

C. Assigning Ranking for Web Pages using Users interest: In links and out-link values are determined updated based on the covariance values. Based on the covariance values Ranking is assigned to the Web Pages.

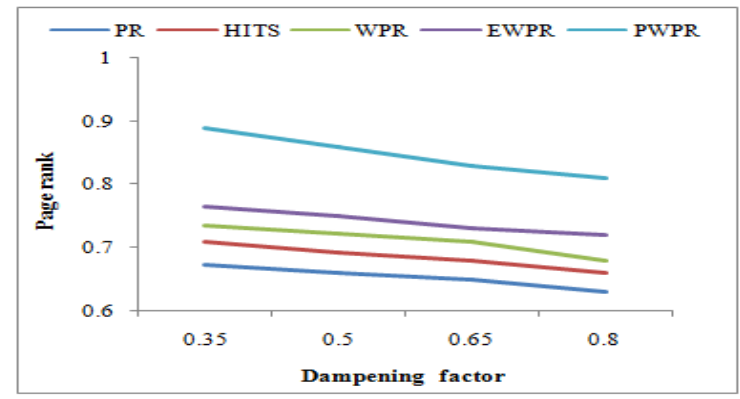

Fig. 4 Comparison of page ranks at $d$

In this section have calculated rank value of each page based on PR, WPR, EWPR, HITS and proposed PWPR algorithm for a web graph shown in Fig. 4.The comparison of results shows the values of page rank using PR, WPR, EWPR, HITS and proposed PWPR algorithm at different $d$ values of $0.35,0.50$ and 0.85 and considering that the value of dampening factor increases, and ultimately the page rank decreases.
D. Recall Value: Recall value is calculated is based on the retrieval of information at true positive prediction, false negative. In general it is defined as

$$
\text { RECALL }=\frac{\text { Truepositive }}{\text { Truepositive+Falsenegative }}
$$

Recall is also known as sensitivity and the information recovery is the fraction of the pages that are related to the query that are successfully retrieved.

$$
R E C A L L=\frac{\mid\{\text { relevant pages }\} \cap\{\text { retrieved pages }\} \mid}{\mid\{\text { relevant pages }\} \mid}
$$

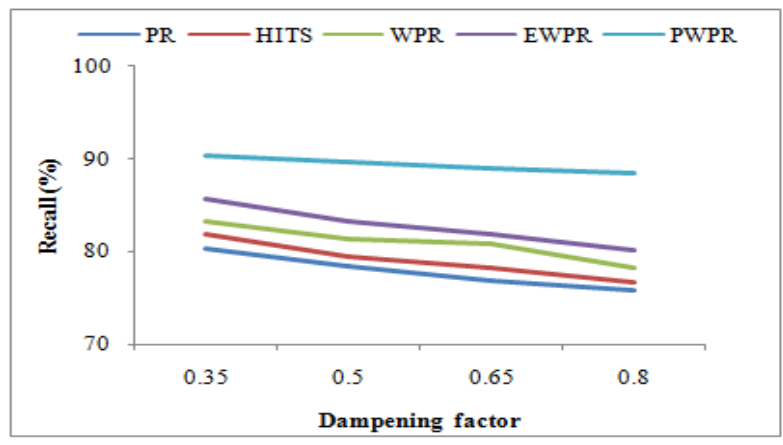

Fig. 5 Comparison of recall at $d$

In Fig.5, the recall values are compared between the PR, WPR, EWPR, HITS and proposed PWPR according to the different dampening parameter ' $d$ '. In this graph, $x$ axis will be the number of dampening factor and $y$ axis will be recall rate. When the numbers of dampening parameter are increased then the recall rate is decreased. This existing system has the lower recall rate compared to the proposed PWPR algorithm. Here weight values are computed using the covariance values from PCA method for page ranks.

E. Mean Average Precision: From the Mean Average Precision and recall are single-value instance based on the complete list of documents returned by the system. For systems that return a ranked sequence of documents, it isdesirable to also consider the order in which the returned documents are presented.Average precision reflects ranking relevant documents higher. It is the average ofprecisions computed at the point of each of the relevant documents in the ranked sequence:

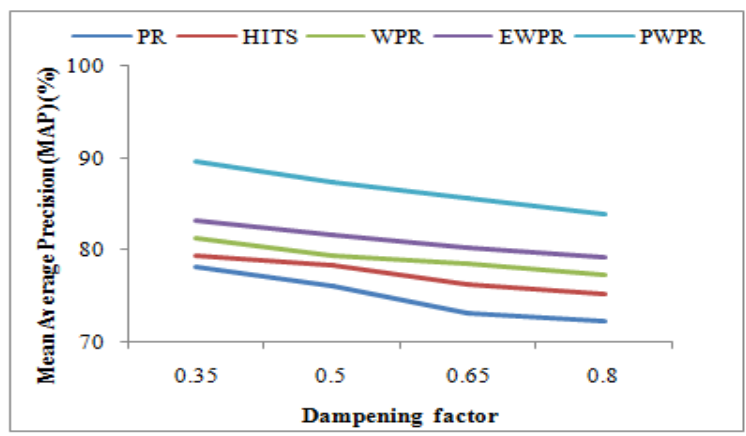

Fig. 6 Comparison of Mean Average Precision (MAP)at $d$ 
In Fig. 6, shows the performance of MAP under different dampening parameter' $d$ ' settings. The results are in Figure 4.PWPRachieves best results when $\alpha=0.96$ and $\mu=20$. The PWPR achieves the best result when $d=0.35$ and $d=$ 0.5 . When $\mathrm{d}$ goes to 0.8 , the PWPR algorithm MAP will reduces and higher than the other schemas. Note that these empirical results show that a small $d=0.35$ and $d=0.5$ is preferred to ensure the effectiveness of PWPR.

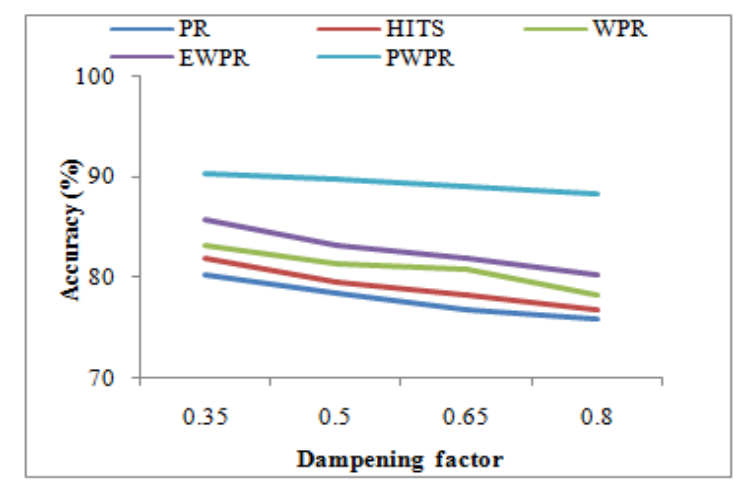

Fig.7 Comparison of ranking accuracy at $d$

In Fig.7, the accuracy values are compared between the PR, WPR, EWPR, HITS and proposed PWPR according to the different dampening parameter' $d$ '. In this graph, $x$ axis will be the number of dampening factor and $y$ axis will be accuracy rate. When the numbers of dampening parameter are increased then the accuracy rate is decreased. This existing system has the lower ranking accuracy rate compared to the proposed PWPR algorithm. Here weight values are computed using the covariance values from PCA method for page ranks.

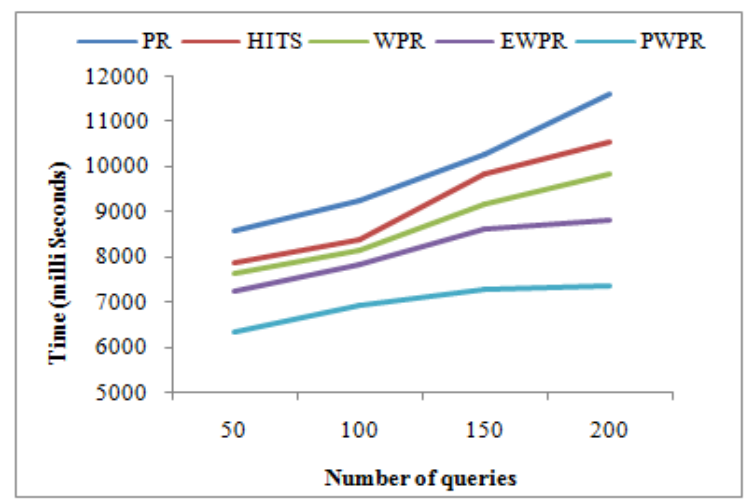

Fig. 8 Comparison of time at number of queries

In Fig. 8, show the time comparison results of different page ranking methods. On number of user queries decrease as the time value is also decreases for PWPR algorithm. This is because the weighted sum of covariance value increases as the ' $d$ ' increases. It can be seen that on data set, PWPR converges faster than EWPR. This is because the average number of out-links of PWPR is larger than that of EWPR and the weight values are smaller on the EWPR when compare to EWPR.

\section{CONCLUSION AND FUTURE WORK}

Thus the user requirement in the webpage is a challenging one due to huge growth in the webpage. In this research a new ranking algorithm for improving the performance of search engines. In the proposed work Weighted Page Ranking (WPR) algorithm is enhanced using Prinicpal Component Analysis (PCA) methods and it is extension to the standard Page Rank algorithm . In this work usually WPR consider the both the inlinks and the outlinks of the webpages and distributes rank scores based on the popularity of the pages.PWPR makes use of number of Visits Of Links (VOL) tocalculate the values of page rank and more relevant results are retrieved first.

Through this method it will the help users toget the relevant information faster manner with accuarcy.PWPR algorithm the page rank of all web pages is being normalizedby using a covariance value from PCA, which reduces the timecomplexity of the conventional page rank algorithm. But in PWPR method new data and updates are constantly arriving, the results of data mining applications become stale and obsolete over time. To overcome thechallenge is that can be done through a Map Reduce (MR) framework is better approach to refreshing mining results for mining big data.Experimental analysis is performed by considering 5000 URL'S it shows that PWPR provides best results in terms of Page rank, MAP, Recall, Accuracy and less execution time to be encouraging. The proposed PWPR algorithm can be adopted by any Search Engine for that this algorithm can be extended for different datasets.

In future, temporal dynamics of user's behaviour can be considered to rank search results to improve its accuracy. In future need to consider some other measures like most recent use of link, information about the user and time spent on web page corresponding to a link.

\section{REFERENCES}

[1] L. Feng, “ Extracting Structure of Web Site Based on Hyperlink Analysis in Wireless Communications, Networking and Mobile Computing", in WiCOM '08, 4th International Conference, 2008.

[2] B. Christophe, V. Verdot, and V.Toubiana, "Searching the'web of things in Semantic Computing" in ICSC Fifth IEEE International Conference, 2011, pp. 308-315.

[3] T. Srivastava, P.Desikan, and V.Kumar, "Web mining-concepts, applications and research directions", Foundations and advances in data mining, Studies in Fuzziness and soft computing, Vol.180, pp. 275-307, Sep 2005.

[4] Q. Zhang, and R.S.Segall, "Web mining: A survey of current research, techniques, and software", International Journal of Information Technology and Decision Making, Vol. 7, No. 04, pp. 683-720, 2008.

[5] B.Singh, and H.K.Singh, "Web data mining research: A survey in Computational Intelligence and Computing Research (ICCIC)", in IEEE International Conference, Dec 2010, pp. 1-10.

[6] W.Xing , and A.Ghorbani, "Weighted PageRank Algorithm", In proceedings of the $2 \mathrm{rd}$ Annual Conference on Communication Networks and Services Research, 2004, pp. 305-314.

[7] A.M.Z. Bidoki and N. Yazdani, “ DistanceRank: An intelligent ranking algorithm for web pages", Information Processing and Management, Vol. 44, No.2, pp. 877-892, 2008. 
[8] T. Abou-Assaleh, T. Das, G. Weizheng, M.Yingbo, P. O'Brien P, and Z. Zhen, "A Link -Based Ranking Scheme For Focused Search" in WWW2007, ACM Press, 2007.

[9] F. Lamberti, A. Sanna, and C. Demartini, "A relation-based page rank algorithm for semantic web search engines", Knowledge and Data Engineering, IEEE Transactions, Vol. 21, No.1, pp. 123-136, 2009.

[10] L.W. Lee, J.Y. Jiang, C. Wu, and S.J. Lee. "A query-dependent ranking approach for search engines", In WCSE'09 Second International Workshop on Computer Science and Engineering, Vol. 1, pp. 259-263, Oct 2009.

[11] Milan Vojnovic, James Cruise, Dianan Gunawardena, and Peter Marbach "Ranking and Suggesting Popular Items", In IEEE Transaction of Knowledge and Data Engineering, Vol. 21, No. 8, Aug 2009.

[12] S. Cheng, P. YunTao, Y. JunPeng, G.Hong, Y.ZhengLu, and H. ZhiYu, "PageRank, HITS and impact factor for journal ranking". WRI World Congress on Computer Science and Information Engineering, Vol. 6, pp. 285-290, 2009.

[13] P. Sharma, Deepak Tyagi and P. Bhadana, "Weighted page content rank for ordering web search result", International Journal of Engineering Science and Technology, Vol. 2, No.12, pp. 7301-7310, 2010.

[14] P. Rani, and E.S.Singh, "An Offline SEO (Search Engine Optimization) Based Algorithm to Calculate Web Page Rank
According to Different Parameters", International Journal of Computers and Technology, Vol. 9, No.1, pp. 926-931, 2013.

[15] Punit Patel, "Research of Page ranking algorithm on Search engine using Damping factor", International Journal of Advance Engineering and Research Development (IJAERD), Vol. 1, No. 1, February 2014.

[16] S. Tuteja, "Enhancement in Weighted Page Rank Algorithm Using VOL", IOSR Journal of Computer Engineering (IOSR-JCE), Vol. 14, No.5, pp. 135-141, 2013.

[17] R. Jain, and D.G. Purohit, "Page ranking algorithms for web mining “, International journal of computer applications, Vol. 13, No.5, pp. 22-25, 2011.

[18] Ruby-Figueroa, R. (2015). Principal Component Analysis (PCA), In Encyclopedia of Membranes, pp.1-2, 2015.

[19] S. Seo, E.J. Yoon, J. Kim, S. Jin, J.S. Kim, and S. Maeng, "Hama: An efficient matrix computation with the map reduce framework", IEEE Second International Conference on Cloud Computing Technology and Science, pp. 721-726, 2010.

[20] L. Fegaras, C. Li, and U. Gupta, "An optimization framework for map-reduce queries", In Proceedings of the 15th International Conference on Extending Database Technology ACM, pp. 26-37, 2012. 